\title{
Study on the Volatility of Stock Price of Suzhou's High-Tech Enterprise by Fractal Analysis Method
}

\author{
Ming Chen \\ School of Business, Soochow University, Suzhou, China \\ E-mail: cm192@163.com
}

Keywords: Fractal Analysis; High Technology; Stock Price; Enterprise

\begin{abstract}
The high-tech enterprises have promoted the transformation and upgrading of modern economy. The author has induced fractal analysis method to make an empirical analysis on the typical case of listed high-tech enterprise in Suzhou, China, and study the structural factors which influence the market pricing of high-tech enterprises. To bring to light the true value of high-tech enterprises, we must separate the trend factor from the cyclical factor. By the fractal analysis of a typical high-tech enterprise: CTSC. This study has revealed that the volatility of stock price of high-tech enterprises appear as a trend motion accompanied with periodic motion. Then, the author has put forward a new method to separate the trend factor from the cyclical factor of the stock price of high-tech enterprises.
\end{abstract}

\section{Introduction}

High-tech enterprises are becoming more and more important in Suzhou, China. They have promoted the transformation and upgrading of economy, and become the important strength to promote economic globalization. In the 21st century, the business environment of enterprise changed rapidly.

The "China Hi-tech Industrial Park Foresight and Investment Strategy Analysis Report (20132017) "[1] has show, since the "11th Five-Year Plan", remarkable achievements have been made in the industrialization of China's high and new technologies, and a series of major technological innovations have emerged. The ability of independent innovation is further improved. High speed railways, wireless broadband communications, high performance computers, new energy vehicles and other technologies have entered the world's advanced ranks. Several high and new technology major sets of equipment and their key components have been independently designed and manufactured. Electronic commerce, digital media and other modern service industries are emerging with the support of high and new technologies.

There is less than a quarter-century since the high-tech enterprise appearance in Suzhou, but they have played an important role in the economic development of Suzhou. The high-tech enterprises have raised the level of Suzhou's economy in every field, such as information and space, new materials, advanced manufacturing, energy, advanced transportation and other fields.

The high-tech enterprises have not yet commenced its full course; many features are not yet ripe. So, people can only stay in the perceptual stage, but can not rise to rational cognition.

Especially in the stock market, as new technology enterprises, many of them are based Internet. At the beginning of the Internet Age, people are immersed in an exaggerated imagination, blindly exaggerate the function and value of the internet, and has neglected of its profit creation function. This basic dislocation has taken the wrong road of theory and practice.

When people recognized this point, the stock index of high-tech enterprises fell sharply. They thought that the high-tech enterprises will be unable to get up after the fall. This mood of the most changeful has greatly hindered people to get rational judgment of the development of the high-tech enterprises. 


\section{The METHOD of Fractal Analysis and the Biased Random Walk}

The traditional capital asset pricing model assumes that we live in a no transaction costs, no commission and no tax world. It separates out the investor behavior from their social restraints. Of course this is far from the case. Many empirical studies find that the distribution of stock return deviates significantly from the Gauss distribution, present a kind of "fat tail" characteristic ${ }^{\text {[2]. }}$ The probability in the region of high yield and high loss is greater than the probability of Gauss distribution. That is to say, the hypothesis that market investors are mutually independent or weakly dependent is untenable. The linkage among investors is one of the characteristics of the market structure itself. It reflects the herd behavior in financial markets, and the herd behavior leads to the "fat tail" characteristic ${ }^{[3]}$ of stock return distribution. The unexpected events of financial market not only has multi factor, nonlinear, but also has uncertainty. Some studies show, in the financial markets, people are not equal to digest and confirm information. It often leads to the biased random walk of stock price, or called Fractional Brown Motion (FBM) ${ }^{[4]}{ }^{[5]}$. So, we have no reason to believe that markets must be balanced and effective. Financial market may perfectly possible come to the fractal structure.

The word "Fractal" is proposed for the first time in 1975 by Benoti B.Mandelbrot ${ }^{[6]}$. Its original meaning is "no rules, score, reduced to fragments". The mathematical definition of fractal is: If a set in Euclidean space whose Hausdorff dimension (DU) ${ }^{[7]}$ is always greater than its topological dimension (DT), i.e. DU>DT, we call the set as a "fractal set", and "fractal" for short. Common ground says, thing's components similar to the whole body in a certain way called fractal.

Contrary to the efficient market hypothesis, the financial market is not linear, balanced or orderly, but non equilibrium, nonlinear, and disordered. It is chaotic, and obey fractal Brown motion ${ }^{[8]}$. Rely on the fractal geometry language, we can get a more in-depth understanding of financial market.

The fractal theory of stock market suggests, the stock market is not, at least not always a general equilibrium efficient market. The changes of stock price are with the characteristics of long-term memory of past history. It is not a completely random walk, but a "biased random walk" ${ }^{19]}$.This process is highly dependent on the initial conditions ${ }^{[10]}$. Its direction of change is uncertain. The final price is not a unique price which stay in general equilibrium state, but a stochastic equilibrium price which comes from the polymorphic equilibrium market determined by multi-attractors.

We can analyze the stock price volatility of CTSC by the Rescaled Range Analysis Method (R/S method) founded by H.E. Hurst ${ }^{[11]}$.

Assume a time series $\{x i\}$, the number of observations is $M$. The basic idea of R/S analysis method is: divide the time series $\left\{x_{i}\right\}$ whose length is $M$ into $A$ adjacent subintervals whose length are $N(2 \leq N \leq L) . L$ is the length of maximum subinterval, $A N=M$. Each subinterval is named $I_{a}$, $(a=1,2, \ldots, A)$. Each $x_{i}$ of Each $I_{a}$ is named $x_{i, a},(k=1,2, \ldots, N)$. Assume the means of $\left\{x_{i}\right\}$ of $I_{a}$ is $e_{a}$, We have:

$$
\begin{aligned}
& x_{k, a}=\sum_{i=1}^{k}\left(x_{i, a}-e_{a}\right), \quad k=1,2, \ldots, N \\
& R_{I_{a}}=\max \left\{x_{k, a}\right\}_{1 \leq k \leq N}-\min \left\{x_{k, a}\right\}_{1 \leq k \leq N} \\
& S_{I_{a}}=\sqrt{\frac{\sum_{k=1}^{N}\left(x_{k, a}-e_{a}\right)^{2}}{N}}
\end{aligned}
$$

Where $N$ is the length of subinterval, $x_{k, a}$ is the accumulation deviation of subinterval, $S_{I a}$ is the standard deviation of the subinterval $I_{a}, R_{I a}$ is the range of the subinterval $I_{a}, R_{I a}$ become big with increasing $N$.

In order to compare different time series, Hurst used the standard deviation of the observed value divided by its range, that is: $R$ divided by $S$. He has the following equation:

$$
(R / S)_{N}=\frac{1}{A} \sum_{a=1}^{A}\left(R_{I_{a}} / S_{I_{a}}\right)=(a N)^{H}
$$

In equation (2), $A$ is the constant; $H$ is the Hurst Index, and $0 \leq H \leq 1,2 \leq N \leq L$. 
There are 3 different types of Hurst Index: (1) $0 \leq H \leq 0.5$, (2) $H=0.5$, (3) $0.5 \leq H \leq 1$. According to the statistic knowledge, when $H=0.5$, the original series is a standard random walk. The present will not affect the future, namely, the original series is lack of statistical correlation in long-term. When $0 \leq H \leq 0.5$, the original series is with anti-persistence. It is often called the mean-reverting, that is, the past increment is negative correlation with the increment of the future. If a series is go up in the earlier period, then, it will probably go down in the next period. On the other hand, if it is go down, then in the next period, it will probably go up. When $0.5 \leq H \leq 1$, the original series is with persistence, that is, its past increment is positively related to the incremental of the future. If a series is go up / down in the earlier period, then, it will continue to go up / down in the next period. The larger value of $H$, the less of noise in this series, the series has stronger persistence and more obvious trend. The persistence level can be measured through $H$ minus 0.5 . There are many of persistent series in nature; the stock market is one of them.

Take the logarithm on both ends of the equation (2):

$$
\ln (R / S)_{N}=H \ln (N)+\ln a \quad \mathrm{~N}=2,3, \ldots, \mathrm{L}
$$

The calculation of Hurst index generally has two methods, both based on equation (3). The first method is taking the length of each subinterval in turn based on the length of the sample, for example, $N=50,100, \ldots, M$. Performing the regression by the OLS method according to equation (3), we can get the values of a plurality of $H$. These estimates are associated with $L$ : the length of maximum subinterval, denoted as $H_{L}$. Using $\left\{H_{L}\right\}$ series, the maximum value corresponding to $L$ is the average cycle length. If $H_{L M}=\mathrm{Max} H_{L}$, then the average cycle length is $L_{M}$, the Hurst index is $H_{L M}$.

The second method is taking $L=M$, then compute $H_{M}$ by the first method. The value of $H_{M}$ is the Hurst index. But using this method we are unable to obtain the average cycle length.

For a series, in order to describe the present effects on the future, B.B.Mendelbrot[12] has introduced a correlation measurement index:

$$
C_{M}=2^{(2 H-1)}
$$

In equation (4), $C_{M}$ expresses the correlation during interval $M$. Thus, independent series ( $H$ $=0.5)$ has no correlation; Series with persistence $(H>0.5)$ has positive correlation, and series with anti-persistence $(H<0.5)$ has negative correlation.

To calculate the long-term memory cycle length of the volatility of the stock return rate, we need to calculate the statistic $V$ :

$$
V_{n}=\frac{R S_{n}}{\sqrt{n}}
$$

Where $n$ is the number of days. The interval in which $V$ keeps linear growth is just the long-term memory cycle length. Strictly speaking, the Hurst index should be calculated in the long-term memory time cycle.

\section{Market Performance of a Typical High-Tech Enterprise: CTSC}

In the most influential listed high-tech enterprises in Suzhou, China, the Create Technology \& Science Co. is a typical one. We choose Create Technology \& Science Co. (CTSC) as the typical case. Using its historical stock price data, carry through demonstration analysis by the fractal method, to inquire about the structural factors which influence its stock price movements, and get a deepen understanding of the pricing of the high-tech enterprises.

CTSC is an investment holding company, founded in 1993 and listed on the Shenzhen Stock Exchange in 1994. By the end of 2014, its net assets was of 1 billion 324 million RMB Yuan. CTSC's current share capital is 400080405 shares [13]. CTSC's main business is the R \& D, production and sale of power transmission high-voltage insulators, clean environmental protection equipment and engineering; CTSC also involved in surveying and mapping instruments, abrasives, 
needle roller bearings, and import and export trade [13]. The controlling shareholder of CTSC is Suzhou Chuangyuan Investment Development (Group) Co., Ltd which holds 34.33\% stake of CTSC.

CTSC's daily stock closing price volatility (backward answer authority) from Oct.19, 2009 to Oct. 17, 2017 is shown in figure 1:

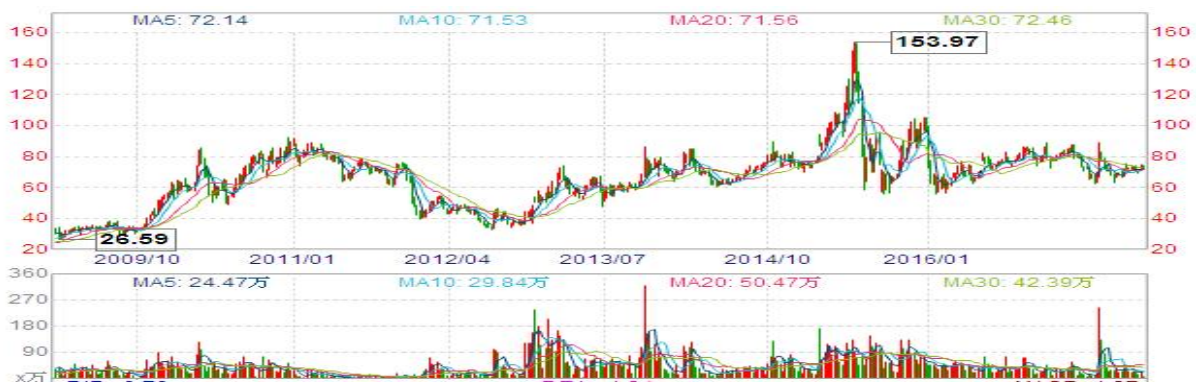

Data Source: http://finance.sina.com.cn/ realstock /company /sz000551/ nc.shtml

Fig. 1. Volatility of CTSC Daily Stock Closing Price (Oct.19, 2009 to Oct. 17, 2017, backward answer authority)

\section{Fractal Analysis of CTSC Stock Price}

In this study, we use the first method to calculate the Hurst index. In the estimation and regression process, we use the logarithmic rate of stock return of CTSC. That is, make:

$$
S_{t}=\ln \left(p_{t} / p_{t-1}\right)
$$

Where $p_{t}$ is the stock price at time $T$; $S_{t}$ is the logarithmic stock return rate at time $T$, For $\mathrm{R} / \mathrm{S}$ analysis, Logarithmic rate of return is more suitable than the percentage change in price. In R/S method, the range is the cumulative deviation to the average value. The sum of logarithmic return rate is equal to the cumulative return rate, while the percentage change is not. The logarithmic stock return rate of CTSC is shown in figure 2:

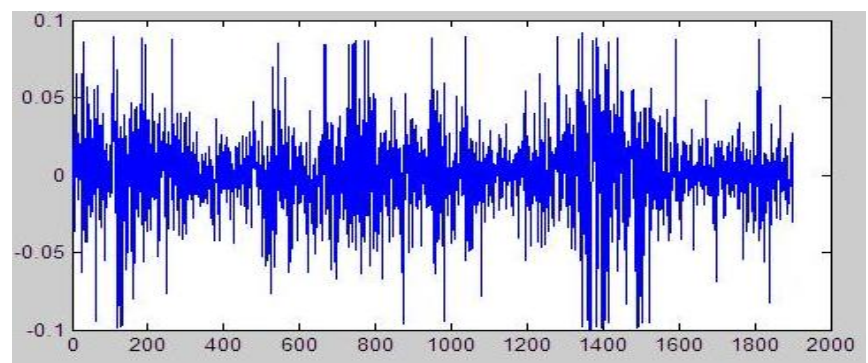

Fig. 2. The logarithmic Stock Return Rate of CTSC

We now explore the probability distribution of the logarithmic stock return rate of CTSC. It is shown in figure3:

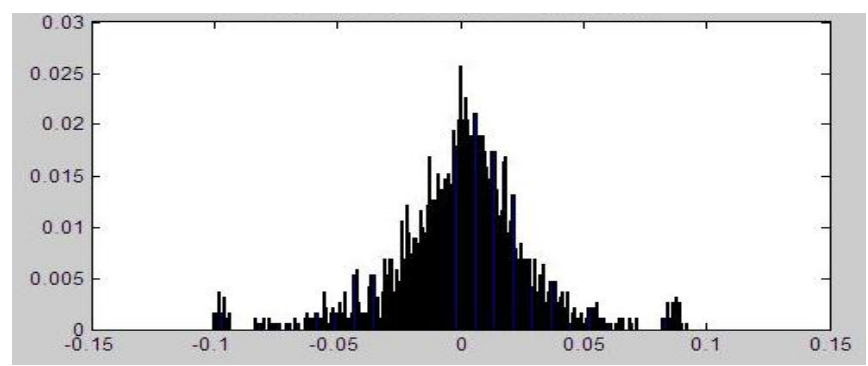

Fig. 3. The Probability Distribution of the Logarithmic Stock Return Rate of CTSC

It can be seen from figure 3 that the tail of the probability distribution of return rate of CTSC is much fatter than the Normal distribution. It is not a completely random walk, but a biased random walk. To further prove this, we do the P-P Normal Distribution Test. The result is shown in figure 4: 


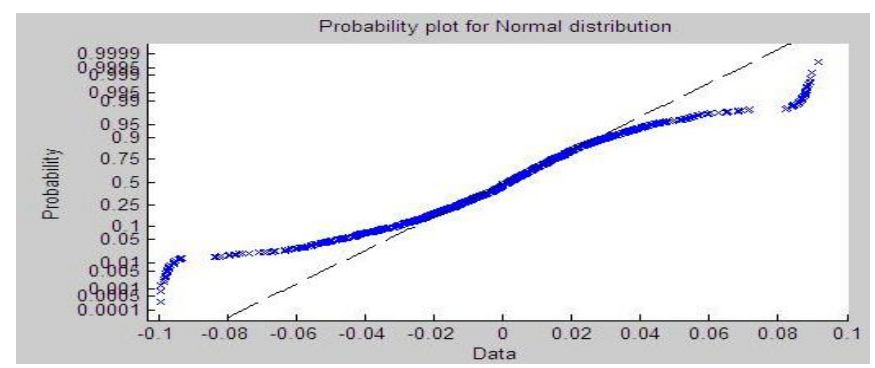

Fig. 4. The P-P Normal Distribution Test of the Logarithmic Stock Return Rate of CTSC

Figure 4 shows, the blue curve is not a straight line, and do not coincide with the dotted line, which proves that the distribution is not a normal distribution. So, the logarithmic stock return rate of CTSC can and should be studied with the fractal method as mentioned above.

Carries on R/S analysis to $\{\mathrm{xt}\}$ series, respectively taking $\mathrm{t}=50,100,150, \ldots, 850$ trading days. Calculating according to the above equations, we got the following $N, \ln N, R / S$, $\ln (R / S)$ statistic table (Tab. 1):

TABLE 1. $N, L n n, R / S, L n(R / S)$ Statistic Table

\begin{tabular}{|c|c|c|c|}
\hline $\mathbf{N}$ & $\mathbf{l n} \mathbf{N}$ & $\mathbf{R} / \mathbf{S}$ & $\ln (\mathbf{R} / \mathbf{S})$ \\
\hline 50 & 3.912 & 7.9359 & 2.0714 \\
\hline 100 & 4.6052 & 12.0342 & 2.4878 \\
\hline 150 & 5.0106 & 14.9151 & 2.7024 \\
\hline 200 & 5.2983 & 18.0337 & 2.8922 \\
\hline 250 & 5.5215 & 16.6205 & 2.8106 \\
\hline 300 & 5.7038 & 20.6929 & 3.0298 \\
\hline 350 & 5.8579 & 21.7415 & 3.0792 \\
\hline 400 & 5.9915 & 23.4851 & 3.1564 \\
\hline 450 & 6.1092 & 26.9047 & 3.2923 \\
\hline 500 & 6.2146 & 28.4511 & 3.3482 \\
\hline 550 & 6.3099 & 30.0869 & 3.3989 \\
\hline 600 & 6.3969 & 27.7538 & 3.3234 \\
650 & 6.477 & 29.7871 & 3.3941 \\
700 & 6.5511 & 32.2834 & 3.4746 \\
750 & 6.6201 & 35.1672 & 3.5601 \\
800 & 6.6846 & 38.1759 & 3.6422 \\
850 & 6.7452 & 37.5627 & 3.626 \\
900 & 6.8024 & 37.0752 & 3.6129 \\
950 & 6.8565 & 38.7443 & 3.657 \\
\hline
\end{tabular}

Draw $\ln N$--- $\ln (R / S)$ line graph, as shown in figure5:

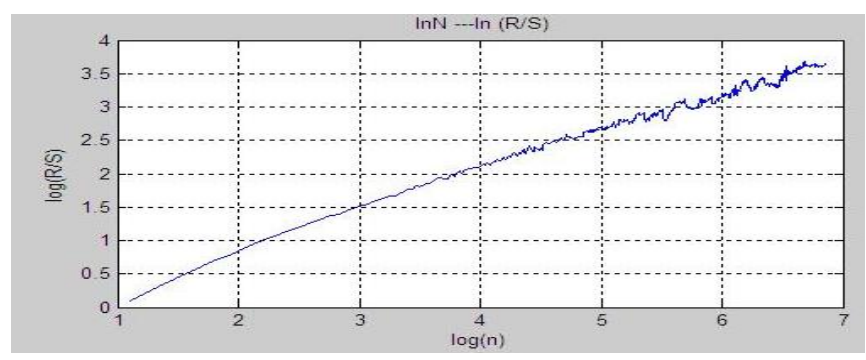

Fig. 5. $\ln N$----ln (R/S) Line Graph

We have calculated the statistic V, as shown in figure 6: 


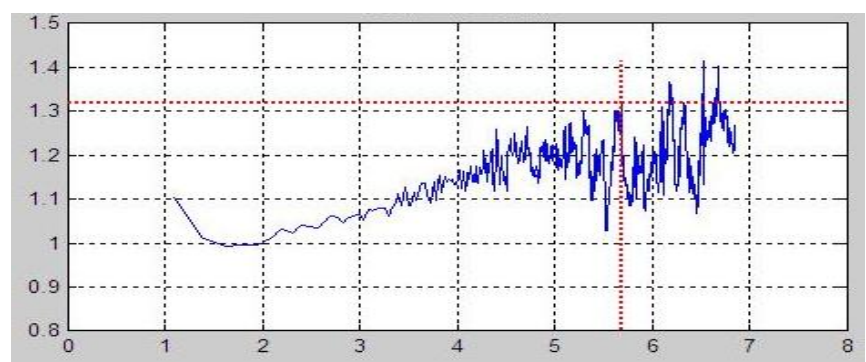

Fig. 6. V and Its Inflection Point of the Logarithmic Stock Return Rate of CTSC

From figure 6, we can find that the Inflection Point of $V$ is 5.6937, and the long-term memory cycle length corresponding to the inflection point of $V$ is 297 days.

Then, going back to figure 5, we perform OLS regression use the data $0 \leq \ln N \leq 5.6937$ to estimate the value of $H$, as shown in figure 7, the result is:

$$
\ln (R / S)=-0.2324+0.5788 \ln N
$$

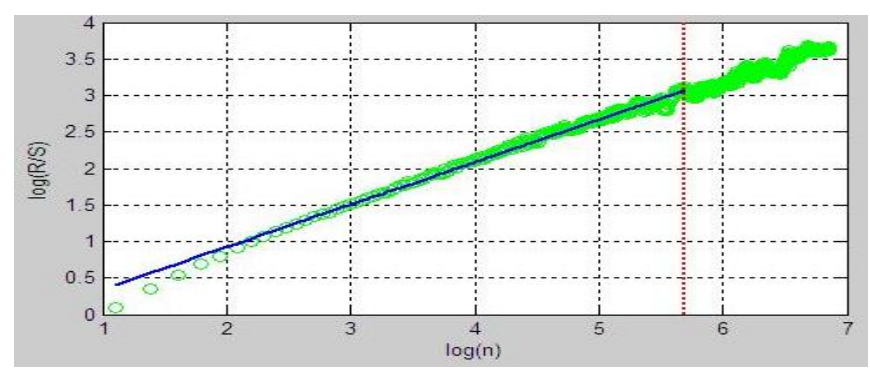

Fig. 7. Linear Fitting Chart of $\ln (\mathrm{RS})$ to $\ln \mathrm{N}(0 \leq \ln \mathrm{N} \leq 5.6937)$

From equation (7), we have obtained the Hurst Index: Hurst=0.5788.

The Regression Statistics and Regression Structure of CTSC is shown in Table 2:

Table 2. The Regression Statistics and Regression Structure of CTSC

\begin{tabular}{|c|c|c|}
\hline $\mathrm{R}^{2}$ & \multicolumn{2}{|c|}{0.9893} \\
\hline $\mathrm{F}$ & \multicolumn{2}{|c|}{27174.5} \\
\hline$P$ & \multicolumn{2}{|c|}{0} \\
\hline Error Variance & \multicolumn{2}{|c|}{0.002950} \\
\hline \multirow{2}{*}{ Triangular Factor } & -83.18 & -16.9333 \\
\hline & 0 & -3.2039 \\
\hline $\mathrm{df}$ & \multicolumn{2}{|c|}{295} \\
\hline normr & \multicolumn{2}{|c|}{0.9328} \\
\hline
\end{tabular}

Regress results shows, $H=0.5788$. The higher $\mathrm{R}^{2}$ (0.9893), higher $\mathrm{F}$ value (27174.5) show a good fitting degree of this regression.

Through the value of $H$, we can calculate the value of correlation metric index $C_{M}$ is 1.1156 . It indicates that the volatility of CTSC stock price has fractal structure and persistence to a certain degree. It is not a random walk process, but a biased random walk process. However, the $H$ value is not deviate greatly from 0.5, also show that there exist large noise components. Different from the ordinary random walk process, the stock return series is a biased random walk process, because it has a "long-term memory" in play. The long-term memory is not infinite, but is limited. According to the calculation above, the average long-term memory cycle of stock price volatility of CTSC is about 297 trading days. R/S analysis shows that, in the long-term memory cycle, the volatility of stock return rate of CTSC is a persistent series with fractal probability distribution. It follows a biased random walk process. The market shows some tendency strengthens behavior, rather than the mean reverting behavior, but there are still large noise components in it. When exceeding the long-term memory cycle, the volatility of stock return rate is no longer reflected the tendency strengthens behavior. 


\section{REGRESS Analysis of CTSC Stock Price}

Based on the analysis above, we then analyze the volatility of stock return rate of CTSC Inc. by traditional econometric. Now that the long-term memory cycle of CTSC stock price volatility is about 300 trading days, we perform the quasi linear fitting using a linear function and a Sine function for the cycle of 300 days of time, That is, we perform the following regression analysis:

$$
p=a+b t+\sum_{i=0}^{5} c_{i} \sin \left[2 \pi t /\left(297 \times 2^{i}\right)\right]
$$

By regression calculation, we have obtained the following parameter values:

$$
\begin{aligned}
& \mathrm{p}=56.1018+5.9045 \mathrm{t}-2.2462 \sin (2 \pi \mathrm{t} / 297)-2.0301 \sin [2 \pi \mathrm{t} /(297 \times 2)]+18.4168 \sin [2 \pi \mathrm{t} /(297 \times 4)]- \\
& 139.5989 \sin [2 \pi \mathrm{t} /(297 \times 8)]+2522.7268 \sin [2 \pi \mathrm{t} /(297 \times 16)]-13456.9512 \sin [2 \pi \mathrm{t} /(297 \times 32)](9)
\end{aligned}
$$

The main statistics are shown in Table 3:

Table 3. Main Statistics of the Quasi Linear Regression of CTSC Stock Price

\begin{tabular}{|c|c|c|c|}
\hline $\mathrm{R}^{2}$ & $\mathrm{~F}$ & $\mathrm{P}$ & Error Variance \\
\hline 0.4892 & 259.27 & 0 & 112.96 \\
\hline
\end{tabular}

The fitting curve is shown in figure 8:

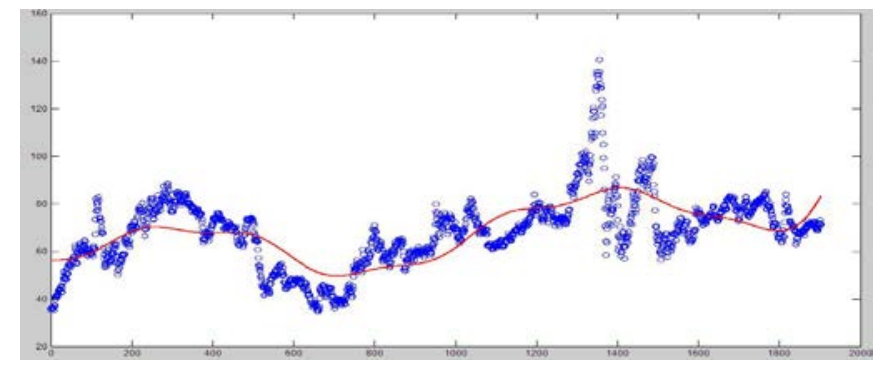

Fig. 8. The fitting Curve of the Quasi Linear Fitting Regression

\section{Conclusions}

We can see that the volatility of stock return rate of high-tech enterprises appears as a weak trend with cyclical fluctuations. In a large time span, the predictive value is good agreement with the actual value. Through the calculation above, for a typical high-tech enterprises company in Suzhou: CTSC, We have found that its stock price took 56.11 RMB Yuan (about US \$9.35) as the benchmark, then, move in cycles based on a 297 trading day cycle.

Through the R/S analysis and regression analysis, equation (9) has achieved the separation of the trend motion and periodic motion of the stock price of high-tech enterprises. The linear rising trend may be related with the development of high tech economy. The violent fluctuation of their stock price is mainly derived from the cyclical motion of the development of economy. However, the cyclical motion of the volatility of the stock price is often due to the violent fluctuation of investors' emotion. We have found that, the sharp fluctuations of stock price of hightech enterprises is the reaction of the changes of capital market's enthusiasm to a great degree, rather than the really change of their intrinsic value. To bring to light the true value of hightech enterprises, we must separate the trend factor from the cyclical factor of the volatility of their stock price. Our study has put forward a new method for this separation.

\section{Acknowledgment}

Thanks for the Philosophy and Social Sciences Foundation of Jiangsu, China (EYB022). 


\section{References}

[1] Foresight Industry Research Institute, China Hi-tech Industrial Park Foresight and Investment Strategy Analysis Report (2013-2017) [Z], Baidu Wenku,2014,5

[2] Chiu C W, Mumtaz H, Pintér G. Forecasting with VAR models: Fat tails and stochastic volatility [J]. International Journal of Forecasting, 2017.

[3] Aïdékon E. Speed of the biased random walk on a Galton-Watson tree [J]. Probability Theory and Related Fields, 2014, 159(3-4):597-617.

[4] Xu Y, Guo R, Liu D, et al. Stochastic Averaging Principle for Dynamical Systems with Fractional Brownian Motion[J]. Discrete and Continuous Dynamical Systems - Series B (DCDS-B), 2017, 19(4):1197-1212.

[5] Mihajlovsky O. Fractal Characteristics of World Market Commodity Derivatives [J]. Annales Universitatis Apulensis Series Oeconomica, 2013.

[6] Mandelbrot B, B M B, Mandelbrot B B, et al. The Fractal Geometry of Nature [J]. The Fractal Geometry of Nature, 1982.

[7] Goldberger A L, Amaral L A N, Hausdorff J M, et al. Fractal dynamics in physiology: Alterations with disease and aging[J]. Proc Natl Acad Sci U S A., 2002, 99(4):2466-2472.

[8] Li J, Huang J. Dynamics of 2d Stochastic Non-Newtonian Fluid Driven by Fractional Brownian Motion [J]. Discrete and Continuous Dynamical Systems - Series B (DCDS-B), 2017, 17(7):24832508.

[9] Taggi L. Absorbing-state phase transition in biased activated random walk [J]. Electronic Journal of Probability, 2016, 21.

[10] A. Croydon D, Fribergh A, Kumagai T. Biased random walk on critical Galton-Watson trees conditioned to survive [J]. Probability Theory and Related Fields, 2013, 157(1-2):453-507.

[11] Hurst H E. The Problem of Long-Term Storage in Reservoirs [J]. International Association of Scientific Hydrology Bulletin, 1956, 1(3):13-27.

[12] Mandelbrot B, B M B, Mandelbrot B B, et al. Fractal character of fracture surfaces of metals [J]. NATURE, 1984, (5961):721-722.

[13] Create Technology \& Science Co. (CTSC) Introduction[Z]. http://www.000551.cn.13414. m8849.cn/web-ia/item/index.asp. July.26, 2017 